\title{
KRAS mutation of extraovarian implants of serous borderline tumor: prognostic indicator for adverse clinical outcome
}

\author{
Tao Zuo ${ }^{1}$, Serena Wong ${ }^{1}$, Natalia Buza and Pei Hui \\ Department of Pathology, Yale University School of Medicine, New Haven, CT, USA
}

\begin{abstract}
In contrast to non-invasive extraovarian implants, invasive implants of ovarian serous borderline tumor/atypical proliferative serous tumor are associated with adverse outcome and have been reclassified as low-grade serous carcinoma. Mutations of KRAS and/or BRAF have been reported in up to $50 \%$ of serous borderline tumor/atypical proliferative serous tumor. We investigated KRAS and BRAF mutation frequencies in the two types of implants of serous borderline tumor/atypical proliferative serous tumor in correlation with clinical outcome. Forty-two implants of serous borderline tumor from 39 patients were included (invasive implants/low-grade serous carcinoma, $n=20$; non-invasive implants, $n=22)$. KRAS mutation was found in 12 of 20 invasive implants $(60 \%)$ and 3 of 22 non-invasive implants (14\%). BRAF V600E mutation was found in 1 of 22 non-invasive implants (5\%) and none in invasive implants $(0 \%)$. Invasive implants were more frequently associated with higher stage disease. Nine of 14 patients (64\%) with KRAS mutation were found to have stage IIIC disease, while 5 of 24 patients $(20 \%)$ without the mutation had stage IIIC disease. Patients with invasive implants had higher recurrence rate compared to those with non-invasive implants (60 vs $14 \%, P=0.0003$, log-rank test) and worse diseasespecific survival $(P=0.0008$, log-rank test). Regardless of the histological subtypes, patients with KRAS mutation positive implants had significantly higher recurrence rate than those without the mutation $(71 \mathrm{vs} 21 \%, P=0.0021$, log-rank test) and an unfavorable disease-specific survival $(P=0.0104$, log-rank test). In conclusion, compared to those with non-invasive implants, patients with invasive implants present with higher stage of the disease, higher recurrence rate and worse survival. KRAS mutation, but not BRAF V600E mutation, is significantly associated with invasive implants of serous borderline tumor. Regardless of the histological subtypes of the implants, KRAS mutation is a significant prognostic indicator for high risk of tumor recurrence and worse disease-specific survival.

Modern Pathology (2018) 31, 350-357; doi:10.1038/modpathol.2017.121; published online 13 October 2017
\end{abstract}

Extraovarian implants are found in $13-30 \%$ of serous borderline tumors/atypical proliferative serous tumors (SBT/APST). ${ }^{1-6}$ It is crucial to correctly categorize tumor implants to either invasive or noninvasive subtype, as the former is associated with adverse clinical outcome and has been reclassified as low-grade serous carcinoma (LGSC) by the 2014 WHO classification. ${ }^{3}$ Although the molecular mechanism underlying the progression of serous borderline tumor/atypical proliferative serous tumor and their implants remains unclear, many studies

Correspondence: Professor P Hui, MD, PhD, Department of Pathology, Yale School of Medicine, BML254B, 310 Cedar Street, New Haven, CT 06510, USA.

E-mail: pei.hui@yale.edu

${ }^{1}$ These authors contributed equally to this work.

Received 30 April 2017; revised 3 August 2017; accepted 3 August 2017; published online 13 October 2017 have suggested the importance of abnormal activation of mitogen-activated protein kinase (MAPK) pathway, as two key components of the pathway, that is, KRAS and BRAF, are frequently mutated in serous borderline tumor/atypical proliferative serous tumor. ${ }^{1,7,8}$ Approximately $50 \%$ of serous borderline tumor/atypical proliferative serous tumor and lowgrade serous carcinoma harbor KRAS and BRAF mutations, and the incidence of $B R A F$ mutations is higher in serous borderline tumor/atypical proliferative serous tumor than that of low-grade serous carcinoma. ${ }^{4,7,8}$ In one study, up to $70 \%$ of low-grade serous carcinomas were found to have KRAS mutation while only small numbers of serous borderline tumor/atypical proliferative serous tumors had detectable mutated KRAS. ${ }^{9}$ Moreover, vast majority of peritoneal implants, non-invasive and invasive, were found to have identical KRAS or BRAF mutations to their corresponding primary ovarian 
serous borderline tumor/atypical proliferative serous tumors, supporting the hypothesis of clonal progression. ${ }^{10}$ However, additional investigations into the roles of KRAS and BRAF mutations in the pathogenesis of extraovarian tumor implants are needed to solidify the theory, and the prognostic values of these mutations have not yet been fully explored. In this investigation, we examined the presence of KRAS exon 2 and BRAF V600E mutations in comparable numbers of invasive and noninvasive implants of serous borderline tumor/atypical proliferative serous tumors in correlation with tumor stage and patient outcome.

\section{Materials and methods}

\section{Study Case Selection and Histological Review}

Forty-two extraovarian implants of serous borderline tumors in 39 patients were included in this study. All cases were retrieved from pathology archives at a single institution. The primary ovarian serous borderline tumors and their extraovarian lesions were histologically reviewed by all authors and reclassified according to the 2014 WHO criteria into 20 invasive implants/low-grade serous carcinoma and 22 noninvasive implants. ${ }^{2,3,6,11}$ Briefly, tumor implants were classified as invasive based one of the following: lowpower destructive growth pattern, presence of micropapillary architecture and tumor cell nests surrounded by retraction artifact in dense fibrotic stroma (Figure 1). A tumor implant without the aforementioned morphologic qualifiers was considered non-invasive. Patient demographics and clinical follow-up data were collected by medical record review. The study was performed under research protocols approved by the Institutional Review Board.

\section{DNA Extraction and Mutational Analysis}

Formalin-fixed, paraffin-embedded blocks containing target lesional tissues were selected from each case. One hematoxylin and eosin-stained section and additional unstained sections were created on glass slides from the paraffin blocks. Areas of target tumor foci were confirmed to constitute at least $5 \%$ of tumor cells upon hematoxylin and eosin-stained slide review and corresponding tissue areas from the unstained slides were scraped with scalpel into microcentrifuge tubes. DNA was extracted by hydrothermal pressure method of simultaneous deparaffinization and lysis of formalin-fixed paraffinembedded tissue followed by conventional column purification to obtain high quality DNA. ${ }^{12}$

KRAS and BRAF mutation analysis by the high sensitive single strand conformation polymorphism (SSCP) was performed according to previously described method. ${ }^{13}$ Briefly, 5-20 ng of extracted DNA was amplified using PCR primers flanking the hotspot mutations of exon 2 of the KRAS gene (forward 5'-GACTGAATATAAACTTGTGG-3' and reverse 5'-CTGTATCAAAGAATGGTCCT-3') and $B R A F$ V600E mutation (forward primer: 5'-CTCTT CATAATGCTTGCTCTGATAGG-3' and reverse primer: 5'-TAGTAACTCAGCAGCATCTCAGG-3'). The reaction was performed in a $50 \mu \mathrm{l}$ solution containing 1x PCR buffer, $0.1 \mathrm{mM}$ dNTP, $1.5 \mathrm{mM} \mathrm{MgCl}_{2}$ and 2.5 units of AmpliTaq Gold DNA polymerase. PCR started with initial denaturation at $95^{\circ} \mathrm{C}$ for $8 \mathrm{~min}$, followed by 35 cycles of denaturation at $94^{\circ} \mathrm{C}$ for $1 \mathrm{~min}$, annealing at $55^{\circ} \mathrm{C}$ for $1 \mathrm{~min}$ and synthesis at $72{ }^{\circ} \mathrm{C}$ for $2 \mathrm{~min}$, and finished by a final extension at $72^{\circ} \mathrm{C}$ for $10 \mathrm{~min}$ (ABI Veriti Thermal Cycler, Applied Biosystem, Foster City, CA, USA). The PCR product was analyzed by single strand conformation polymorphism (SSCP) using MDE non-denaturing gel. Electrophoresis was carried out on ice for $2 \mathrm{~h}$ and $45 \mathrm{~min}$ at $325 \mathrm{~V}$. The SSCP gel was then stained with SYBR Gold (Molecular Probes, Invitrogen, Norwalk, CT, USA) 1:10 000 in TE buffer added for $20 \mathrm{~min}$ and imaged by Biorad GelDoc UV System (BioRad, Hercules, CA, USA). The presence of KRAS or BRAF mutations were determined by comparing the SSCP banding patterns with those of known KRAS mutation or BRAF V600E mutation positive controls (Figure 2).

\section{Statistical Analysis}

Statistical analysis was performed according to the nature of the data, including Fisher exact test (2-tail), Student $t$-test, and Pearson $X^{2}$ test. The statistical significance was determined by setting level of $P<0.05$. The overall survival was measured in months from the day of initial diagnosis to the date when the patient died of the tumor. The date of detection of tumor recurrence was used for measuring disease-free survival. The Kaplan-Meier statistic method was used to generate $P$-value using log-rank test.

\section{Results}

\section{Clinicopathological Characteristics of the Study Cohorts}

The overall patient demographic and clinicopathologic characteristics of the two study cohorts are presented in Table 1. Among 42 implants of serous borderline tumor/atypical proliferative serous tumor, 20 were classified as invasive and 22 were non-invasive, including one implant with indeterminate invasive morphology (Figure 1f, case 3). Three patients had two implants analyzed in this study that either developed concurrently or at tumor recurrence (cases 2, 3, and 4, Table 1). Overall 20 patients had invasive implants and 19 had non-invasive implants.

\section{BRAF and KRAS Mutations Detected by SSCP}

Using the PCR-SSCP detection methods, BRAF mutation analyses were informative in all cases and 

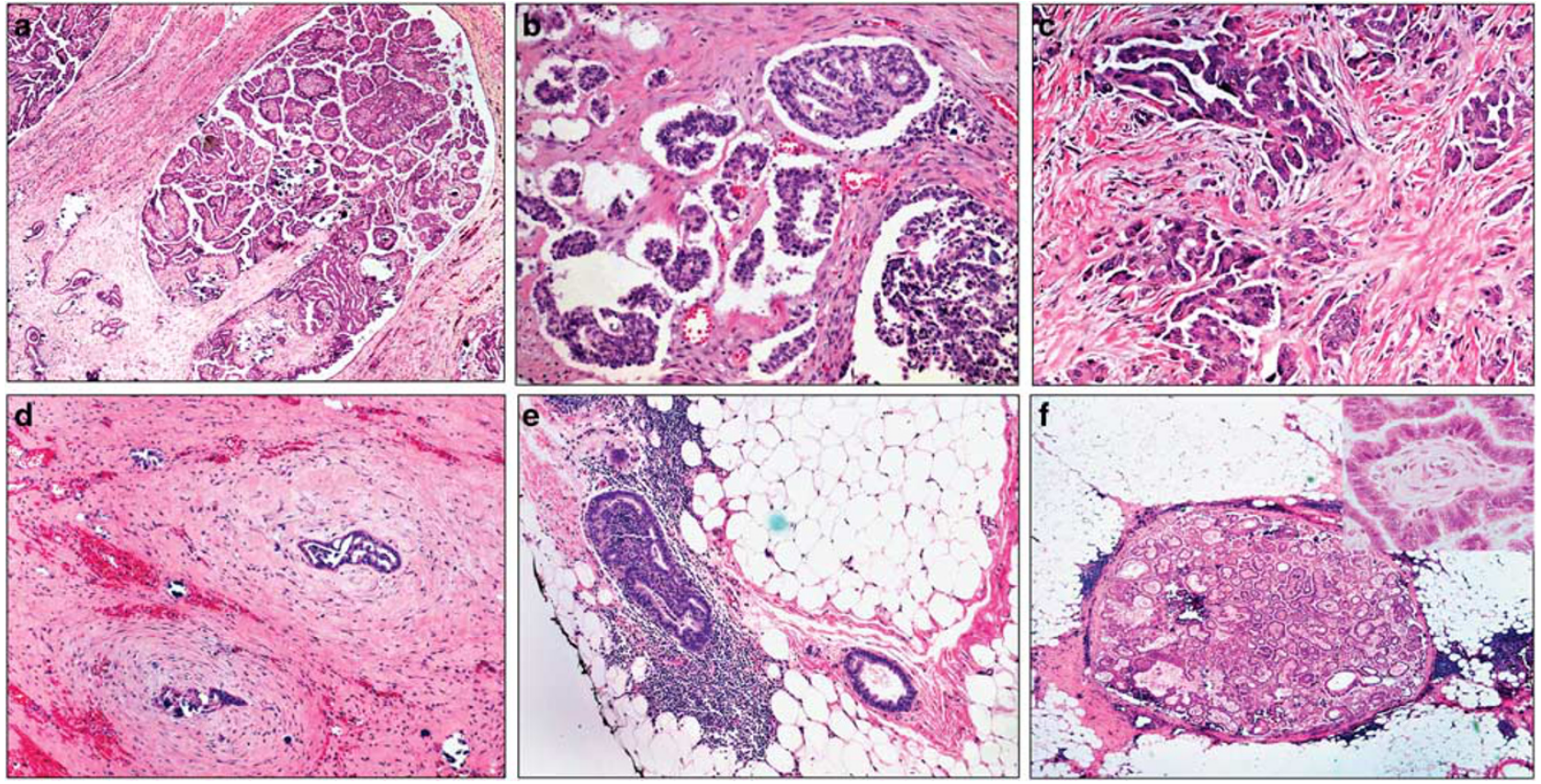

Figure 1 Extraovarian tumor implants of SBT/APST. The tumor implants are classified as invasive based one of the following: low-power destructive growth pattern (a); the presence of micropapillary architecture (b) and tumor cell nests surrounded by retraction artifact in dense fibrotic stroma (c). Non-invasive implants demonstrate either peritoneal surface growth without destructive invasive and without aforementioned features of invasive implants (d,e). Note, the implants presented in $\mathbf{c}$ and $\mathbf{f}$ are from the same patient (case 3, Table 1). The initial tumor implant (f) displayed a well-circumscribed nodule involving the omental adipose tissue as indeterminate implant according to recent criteria. ${ }^{2,6}$

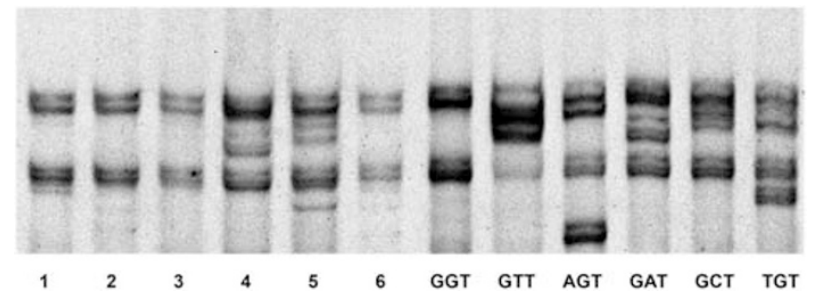

Figure 2 SSCP detection of KRAS mutation in implants of SBT/ APST. Samples 1, 2, 3, and 6 show wild-type KRAS (GGT), while samples 4 and 5 show mutant KRAS, GAT, and GTT, respectively. Samples 7-12 are KRAS wild-type control (GGT) followed by five common KRAS mutation controls (GTT, AGT, GAT, GCT, and TGT).

KRAS test was successful in all but one non-invasive implant sample (case 23, Table 1). As shown in Table 2, KRAS mutation was found in 12 of 20 invasive implants $(60 \%)$ and 3 of 22 non-invasive implants $(14 \%)\left(P=0.0014 ; X^{2}\right.$ test). BRAF V600E mutation was seen in 1 of 22 non-invasive implants $(5 \%)$ and none of the 20 invasive implants $(0 \%)$. PCR-SSCP demonstrated high specificity of calling at 6 genotypes of KRAS at codons 12 and 13, including wild-type GGT and 5 common KRAS mutations, that is, GTT, AGT, GAT, GCT, and TGT (Figure 2). ${ }^{14}$ Among the 15 implants with KRAS mutations, AGT was the most frequent mutation genotype (observed in 5 implants, 33\%). Four implants $(27 \%)$ carried GAT mutation, while GTT (20\%) and GCT (20\%) were each seen in three tumor implants. Only one non-invasive implant showed detectable $B R A F$ V600E mutation.

Invasive tumor implants were more often seen in older patients compared with non-invasive implants (41.0 vs 55.3, $P=0.0016$ ). A similar age distribution was found between patients with KRAS mutation (55.6 years) and those without the mutation (42.7 years, $P=0.0071$ ). In terms of tumor stage, both invasive tumor implants and the presence of KRAS mutation were associated with a higher stage disease (Table 3). However, the laterality of ovarian primary disease was not related to either the morphology or mutation status of implants.

Three patients (cases 2, 3, and 4, Table 1) had two implants analyzed, one invasive and one noninvasive. In case $2, B R A F$ mutation was seen in the non-invasive implant whereas a wild-type $K R A S$ was seen in both the invasive and non-invasive implants. Case 4 had bilateral ovarian serous borderline tumors/atypical proliferative serous tumors with non-invasive implants on the uterine surface (Stage IIA) at the initial staging surgery, and 13 years later the patient developed recurrent disease with both invasive and non-invasive implants, of which KRAS mutation was found only in the invasive implant but not in the non-invasive implant. Case 3 presented initially with an omental implant consisting of a well-circumscribed nodular proliferation of papillary tumor nests without destructive growth (Figure 1f), which may be considered as noninvasive by earlier criteria, ${ }^{3}$ or indeterminate category by the most recent classification. ${ }^{2,6}$ However, 
Table 1 Overall clinicopathologic features and mutation status of study cases $(n=39)$

\begin{tabular}{|c|c|c|c|c|c|c|c|c|c|c|}
\hline$P t \#$ & Age & Laterality & Stage & Implant & KRAS & $B R A F$ & Surgery & Follow-up (mo) & Recurrence & Status \\
\hline 1 & 28 & $\mathrm{R}$ & IIIC & Non-invasive & wt & wt & $\mathrm{RSO} / \mathrm{Tu}$ resec & 168 & No & NED \\
\hline $2^{\mathrm{a}}$ & 58 & $\mathrm{Bi}$ & IIIB & $\begin{array}{l}\text { Invasive } \\
\text { Non-invasive }\end{array}$ & $\begin{array}{l}\text { wt } \\
\text { wt }\end{array}$ & $\begin{array}{l}\text { wt } \\
\text { mut }\end{array}$ & TAH/BSO staging & 144 & No & NED \\
\hline $3^{\mathrm{b}}$ & 52 & $\mathrm{Bi}$ & IIIC & $\begin{array}{l}\text { Invasive } \\
\text { Indeterminate }\end{array}$ & $\begin{array}{l}\text { mut (GTT) } \\
\text { mut (GTT) }\end{array}$ & $\begin{array}{l}\text { wt } \\
\text { wt }\end{array}$ & TAH/BSO staging & 151 & Yes & DOD \\
\hline $4^{\mathrm{C}}$ & 39 & $\mathrm{Bi}$ & IIA & $\begin{array}{l}\text { Non-invasive } \\
\text { Invasive }\end{array}$ & $\begin{array}{l}\text { wt } \\
\text { mut (GCT) }\end{array}$ & $\begin{array}{l}\text { wt } \\
\text { wt }\end{array}$ & TAH/BSO staging & 186 & Yes & DOD \\
\hline 5 & 55 & $\mathrm{Bi}$ & IIIA & Invasive & wt & wt & TAH/BSO staging & 24 & Yes & DOD \\
\hline $6^{\mathrm{d}}$ & 48 & $\mathrm{n} / \mathrm{a}$ & IIIA & Non-invasive & wt & wt & Tumor excision & 48 & No & NED \\
\hline 7 & 66 & $\mathrm{Bi}$ & IIIA & Invasive & mut (GCT) & wt & TAH/BSO staging & 10 & No & NED \\
\hline $8^{\mathrm{e}}$ & 38 & $\mathrm{Bi}$ & IIIB & Invasive & mut (AGT) & wt & TAH/BSO staging & 60 & No & NED \\
\hline $9^{e}$ & 47 & $\mathrm{Bi}$ & IIIC & Invasive & wt & wt & TAH/BSO staging & 58 & Yes & DOD \\
\hline 10 & 46 & $\mathrm{Bi}$ & IIIC & Invasive & wt & wt & TAH/BSO staging & 24 & No & NED \\
\hline 11 & 41 & $\mathrm{Bi}$ & IIIB & Invasive & wt & wt & TAH/BSO staging & 60 & No & NED \\
\hline $12^{\mathrm{e}}$ & 55 & $\mathrm{Bi}$ & IIIC & Invasive & mut (AGT) & wt & TAH/BSO staging & 136 & Yes & AWD \\
\hline 13 & 58 & $\mathrm{Bi}$ & IIIC & Invasive & mut (GTT) & wt & TAH/BSO staging & 52 & Yes & DOD \\
\hline 14 & 75 & $\mathrm{Bi}$ & IIIC & Invasive & mut (AGT) & wt & TAH/BSO staging & 24 & Yes & DOD \\
\hline 15 & 26 & $\mathrm{R}$ & IIIC & Non-invasive & wt & wt & TAH/BSO staging & 52 & No & NED \\
\hline 16 & 51 & $\mathrm{Bi}$ & IIIC & Invasive & mut (AGT) & wt & TAH/BSO staging & 54 & Yes & DOD \\
\hline $17^{\mathrm{d}}$ & 72 & $\mathrm{n} / \mathrm{a}$ & IIIC & Invasive & mut (GAT) & wt & Tumore debulking & 96 & Yes & DOD \\
\hline 18 & 43 & $\mathrm{R}$ & IIA & Invasive & mut (GCT) & wt & TAH/BSO & 205 & Yes & AWD \\
\hline 19 & 68 & $\mathrm{Bi}$ & IIIC & Invasive & wt & wt & TAH/BSO staging & 42 & Yes & DOD \\
\hline 20 & 77 & $\mathrm{Bi}$ & IIIA & Non-invasive & wt & wt & TAH/BSO staging & 48 & No & NED \\
\hline 21 & 34 & $\mathrm{Bi}$ & IIIB & Non-invasive & wt & wt & TAH/BSO staging & 132 & No & NED \\
\hline 22 & 41 & $\mathrm{Bi}$ & IIB & Non-invasive & wt & wt & TAH/BSO staging & 168 & No & NED \\
\hline 23 & 65 & $\mathrm{Bi}$ & IIIB & Non-invasive & failed & wt & TAH/BSO staging & 184 & No & DUC \\
\hline $24^{f}$ & 34 & $\mathrm{Bi}$ & IIIA & Non-invasive & wt & wt & TAH/BSO staging & 216 & Yes & AWD \\
\hline 25 & 61 & $\mathrm{Bi}$ & IIIC & Invasive & mut (GAT) & wt & TAH/BSO staging & 5 & No & NED \\
\hline 26 & 23 & $\mathrm{Bi}$ & IIIB & Non-invasive & wt & wt & BSO & 336 & No & NED \\
\hline $27^{\mathrm{e}}$ & 47 & $\mathrm{Bi}$ & IIIC & Non-invasive & mut (GAT) & wt & TAH/BSO staging & 133 & Yes & DOD \\
\hline $28^{\mathrm{d}}$ & 60 & $\mathrm{n} / \mathrm{a}$ & IIIC & Invasive & mut (AGT) & wt & Tumor debulking & 180 & Yes & DOD \\
\hline 29 & 37 & $\mathrm{Bi}$ & IIB & Non-invasive & wt & wt & TAH/BSO staging & 73 & No & NED \\
\hline 30 & 26 & $\mathrm{R}$ & IIIA & Non-invasive & wt & wt & RSO & 60 & Yes & DUC \\
\hline 31 & 30 & $\mathrm{~L}$ & IIB & Non-invasive & wt & wt & LSO & 94 & No & NED \\
\hline 32 & 55 & $\mathrm{Bi}$ & IIIB & Non-invasive & wt & wt & TAH/BSO staging & 80 & No & NED \\
\hline 33 & 67 & $\mathrm{~L}$ & IIB & Invasive & wt & wt & TAH/BSO & 48 & No & NED \\
\hline 34 & 65 & $\mathrm{Bi}$ & IIB & Non-invasive & mut (GAT) & wt & TAH/BSO staging & 52 & No & NED \\
\hline 35 & 54 & $\mathrm{Bi}$ & IIIB & Invasive & wt & wt & TAH/BSO staging & 48 & No & NED \\
\hline 36 & 15 & $\mathrm{Bi}$ & IIIA & Non-invasive & wt & wt & TAH/BSO staging & 108 & No & NED \\
\hline 37 & 31 & $\mathrm{Bi}$ & IIIA & Non-invasive & wt & wt & TAH/BSO staging & 252 & No & NED \\
\hline 38 & 37 & $\mathrm{~L}$ & IIIA & Non-invasive & wt & wt & TAH/BSO staging & 57 & No & NED \\
\hline 39 & 35 & $\mathrm{Bi}$ & IIIA & Non-invasive & wt & wt & TAH/BSO & 384 & No & NED \\
\hline
\end{tabular}

Abbreviations: AWD, alive with disease; Bi, bilateral; DOD, dead of disease; DUC, dead of unrelated cause; L, left; LSO, left salpingo-oophorectomy; mo, month; mut, mutant; n/a, unknown; NED, no evidence of disease; Pt, patient; R, right; RSO, right salpingo-oophorectomy; TAH/BSO, total abdominal hysterectomy/bilateral salpingo-oophorectomy; wt, wild type.

${ }^{\mathrm{a}}$ Case 2 had invasive implants at initial staging surgery and after 60 days, developed small bowel obstruction with non-invasive implant on the surface of resected small bowel.

${ }^{\mathrm{b}}$ Case 3 had an indeterminate implant (Figure 1f) and the patient had recurrence with invasive implant (Figure 1c) 13 years later.

${ }^{\mathrm{c}}$ Case 4 had non-invasive implants on the uterine surface (Stage IIA) at the initial staging surgery, and later the patient developed recurrent disease with both invasive and non-invasive implants.

${ }^{\mathrm{d}}$ Cases 6, 17, and 28 had prior surgical history of TAH/BSO for unknown primary tumors, and the laterality of primary ovarian disease can not be determined.

${ }^{\mathrm{e}}$ Cases \#8, 9, 12, and 27 had micropapillary morphological features in either primary ovarian tumors or extraovarian implants.

${ }^{\mathrm{f}}$ Cases \#24 had KRAS (G12V) in primary ovarian tumor detected by next-generation sequencing assay.

Table 2 Histological subtypes of implants and KRAS and BRAF mutation status $(n=42)$

\begin{tabular}{lcccc}
\hline Implant type & Total & KRAS mutation n(\%) & BRAF mutation n(\%) & Wild type n(\%) \\
\hline Non-invasive & 22 & $3(14 \%)$ & $1(5 \%)$ & $17(77 \%)$ \\
Invasive & 20 & $12(60 \%)$ & $0(0 \%)$ & 0.0014 \\
\hline
\end{tabular}

KRAS mutation analysis failed in one non-invasive tumor implant.

ancluding one implant of indeterminate type (case 3, Table 1). 
Table 3 Statistical analyses of the patient cohorts $(N=39)$

\begin{tabular}{|c|c|c|c|c|c|c|}
\hline & Non-invasive $(\mathrm{n}=19)$ & Invasive $(\mathrm{n}=20)$ & $P$-value & KRAS mutant ${ }^{\mathrm{a}}(\mathrm{n}=14)$ & Wild type $(\mathrm{n}=24)$ & $\mathrm{P}$-value \\
\hline Age (mean) & 41.0 & 55.3 & 0.0016 & 55.6 & 42.7 & 0.0071 \\
\hline \multicolumn{7}{|l|}{ FIGO stage } \\
\hline IIA & $0(0 \%)$ & $2(10 \%)$ & 0.0207 & $2(14 \%)$ & $0(0 \%)$ & 0.0259 \\
\hline IIB & $4(21 \%)$ & $1(5 \%)$ & & $1(7 \%)$ & $4(17 \%)$ & \\
\hline IIIA & $8(42 \%)$ & $2(10 \%)$ & & $1(7 \%)$ & $9(38 \%)$ & \\
\hline $\mathrm{IIIB}^{\mathrm{b}}$ & $4(21 \%)$ & $4(20 \%)$ & & $1(7 \%)$ & $6(25 \%)$ & \\
\hline IIIC & $3(16 \%)$ & $11(55 \%)$ & & $9(64 \%)$ & $5(20 \%)$ & \\
\hline Bilateral ovarian disease & $13(68 \%)$ & $16(80 \%)$ & $>0.05$ & $11(79 \%)$ & $17(71 \%)$ & $>0.05$ \\
\hline Recurrence & $3(14 \%)$ & $12(60 \%)$ & 0.0003 & $10(71 \%)$ & $5(21 \%)$ & 0.0021 \\
\hline Follow-up months (median) & $48-384(132)$ & $5-186(56)$ & 0.0008 & $5-186(75)$ & $24-384(87)$ & 0.0104 \\
\hline
\end{tabular}

a The same KRAS mutation was detected in two implants in one patient (Case 3, Table 1). ${ }^{\mathrm{b}} \mathrm{KRAS}$ mutation analysis failed in one IIIB stage case with non-invasive tumor implant. (Case 23, Table 1).

the patient developed recurrence 13 years later with unequivocal invasive implants (Figure 1c). Nonetheless, identical GTT mutation at codon 12 of KRAS was seen in both the initial omental implant and the invasive implant at recurrence (Table 1).

\section{Evaluation of Prognostic Value of Mutation Status of Tumor Implants}

Overall, the study cohort had a median follow-up of 87 months with intervals ranging between 5 to 384 months (Table 3). Twenty patients with invasive implants had a significantly higher recurrence rate $(12 / 20,60 \%)$ compared with those with noninvasive implants (3 of $22,14 \%)(P=0.0003$, logrank test) (Figure 3a). Independent of implant histological subtypes, patients carrying KRAS mutation in their implants had a significantly higher recurrence rate than those without the mutation (71 vs $21 \%, P=0.0021$, log-rank test) (Figure 3b). Among 5 recurrent cases without detectable KRAS mutation in the extraovarian implant, one of cases (Table 1) was found to carry KRAS (G12V) in primary ovarian tumor by next-generation sequencing assay at a commercial laboratory, and in another case the primary ovarian tumor had micropapillary architecture and stage IIIC ovarian borderline tumor.

As expected, patients with invasive implants had a worse disease-specific survival than those with noninvasive implants $(P=0.0008, \quad$ log-rank test; Figure 4a). Regardless of the histological types of implants, the presence of KRAS mutation was also associated with an unfavorable disease-specific survival $(P=0.0104$, log-rank test; Figure 4b). Two patients had GTT $(\mathrm{G} 12 \mathrm{~V})$ mutation and both died of the disease. Three of the five patients with AGT (G12S) mutation also died of their disease.

\section{Discussion}

One of most challenging diagnostic issues of serous borderline tumors/atypical proliferative serous tumors is the histological classification of the

\section{a Disease Free Survival}
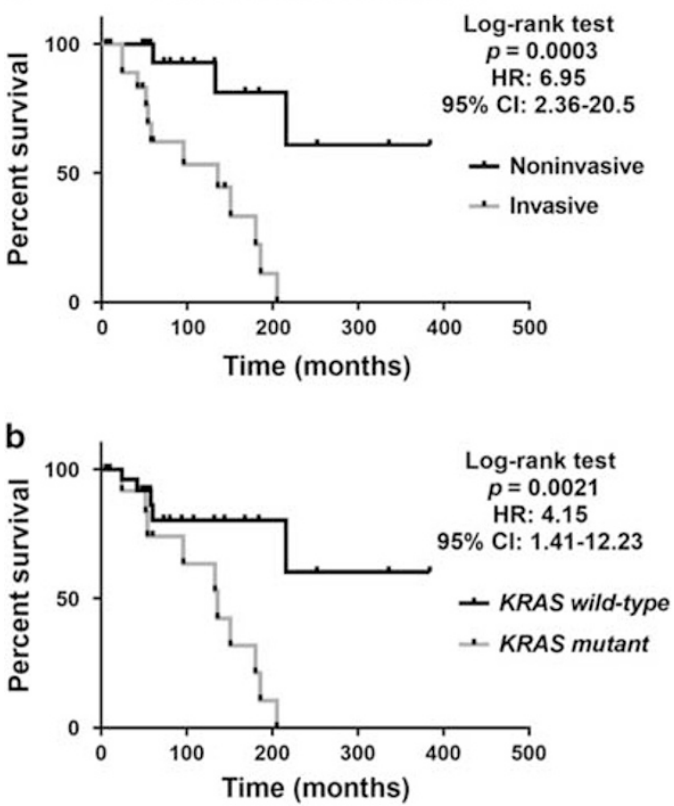

Figure 3 Disease-free survival analyses. (a) Patients with invasive implants (gray) had a higher recurrence rate compared with those with non-invasive implants (black). (b) Independent of implant subtypes, patients with implants harboring KRAS mutations (gray) had a significantly higher recurrence rate than those without KRAS mutation (black).

extraovarian tumor involvement into invasive or non-invasive implants. Precise classification of implants is crucial for patient management and prognostication. In contrast to non-invasive implants, invasive implants of serous borderline tumors/atypical proliferative serous tumors are associated with adverse outcome and are currently reclassified as low-grade serous carcinoma by the 2014 WHO classification. ${ }^{3}$ However, diagnostic separation of extraovarian disease of serous borderline tumors/atypical proliferative serous tumors into invasive and non-invasive implants can be difficult solely based on histological features. ${ }^{11,15,16}$ Recently investigations of large cohorts of serous borderline 
a Disease Specific Survival
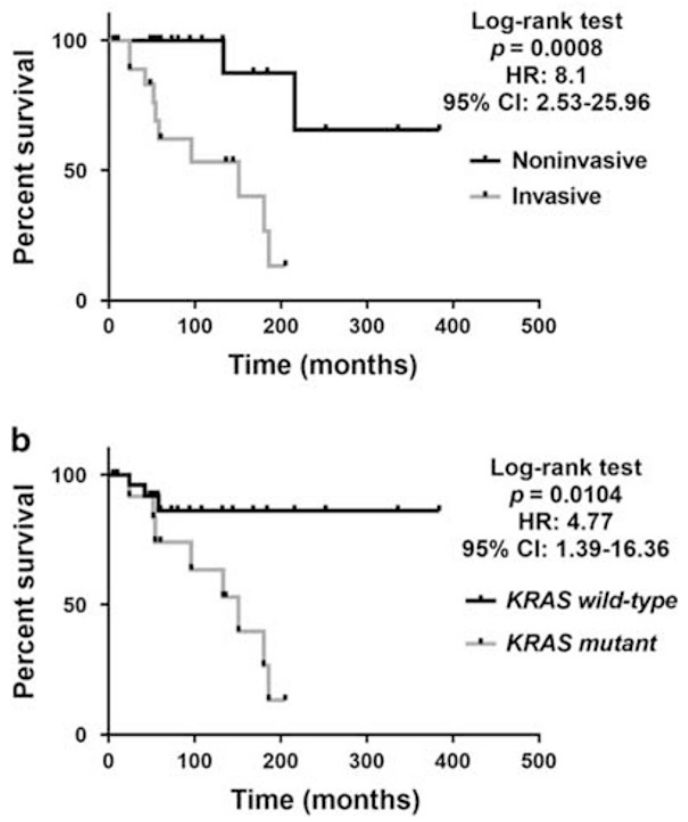

Figure 4 Disease-specific survival analyses. (a) Patients with invasive implants (gray) had a worse overall survival compared with those with non-invasive implants (black). (b) Independent of implant subtypes, patients with implants harboring KRAS mutations (gray) had an unfavorable overall survival compared with those without KRAS mutation (black).

tumors/atypical proliferative serous tumors with extraovarian involvement found that among various histological parameters, destructive invasion of underlying normal tissue, micropapillary architecture, and solid epithelial nests surrounded by clefts should be classified as 'invasive,' whereas those lacking all three features were classified as 'noninvasive. ${ }^{2,11}$ The new WHO classification formally advocates using these histological criteria for classifying such extraovarian lesions of serous borderline tumors/atypical proliferative serous tumors as invasive implants/low-grade serous carcinoma. ${ }^{2,3,11}$ Accordingly, the pattern of destructive growth was considered the most important morphologic feature of invasive implant, and micropapillary architecture and tumor cell nests surrounded by retraction artifact in dense fibrotic stroma were used as the next critical criteria for classifying invasive implants in our study. Corroborating the existing data, our data confirm that invasive implants are more frequently associated with high stage disease (stage IIIC) and higher recurrence rates, and have less favorable overall survival compared with noninvasive implants.

The origin of tumor implants remains enigmatic: independently developed process vs metastatic or clonally derived lesions from the primary tumor. Recent molecular genetic studies have suggested that tumor implants represent clonal extension/spread from the primary ovarian serous borderline tumors/ atypical proliferative serous tumors, as the tumor cells from both sites share the same genetic alterations such as BRAF and KRAS mutations. ${ }^{4,9,10,17}$ Approximately $50 \%$ of serous borderline tumors/ atypical proliferative serous tumors and low-grade serous carcinoma harbor $K R A S$ and $B R A F$ mutations, and the incidence of $B R A F$ mutations is higher in serous borderline tumors/atypical proliferative serous tumors than that of low-grade serous carcinoma. $^{4,7,8}$ Although it is not clear if extraovarian tumor implants were subjected to mutational analysis in every investigation, various studies have shown similar KRAS mutations rates ranging from 57 to $78 \%$ in low-grade serous carcinoma or advanced stage serous borderline tumors/atypical proliferative serous tumors with invasive implants. ${ }^{4,9,10}$ In our study, KRAS and BRAF mutation status were simultaneously analyzed in comparable cohorts of two subtypes of extraovarian tumor implants. Consistent with the literature, KRAS mutation is significantly more associated with invasive implants than non-invasive ones (60 vs 14\%). Of the two patients (cases 2 and 4, Table 1) who each had two implants undergoing mutation analysis (one invasive and one non-invasive), KRAS mutation was seen only in the invasive implant but not in the noninvasive implant in one patient; while the other patient had BRAF mutation present only in the noninvasive implant but wild-type KRAS gene in both implants. One patient (case 3, Table 1) presented initially with a nodular, well-circumscribed implant involving omental adipose tissue (Figure 1f) without destructive growth. While interpreted as noninvasive implant according to the original pathology report, the lesion was reclassified in the current study as indeterminate implant according to recent publications. $^{2,6}$ Interestingly, the patient developed unequivocal invasive implants 13 years later (Figure 1c) and identical KRAS mutation was detected in both the original omental implant and invasive implants at recurrence, consistent with clonal tumor progression.

Interestingly, one recent long-term populationbased study demonstrated that even non-invasive implants carry the statistically significant risk for subsequent development of carcinoma. ${ }^{18}$ Noninvasive implants in our study had a low frequency $(14 \%)$ of KRAS mutation rate. While a similar low rate of KRAS mutation was observed in a prior study $(23 \%),{ }^{8}$ one recent study found that 28 of 55 noninvasive implants $(51 \%)$ had KRAS mutation, ${ }^{10}$ which is even higher than the previously reported mutation rates (17-39.5\%) in serous borderline tumors/atypical proliferative serous tumors and falls into the range of low-grade serous carcinoma (19$54.5 \%){ }^{4}$ It is worth noting that among 55 patients examined in that study, ${ }^{10} 5$ patients had foci of lowgrade serous carcinoma at the primary tumor sites and 4 patients also had concurrent invasive implants. It is possible that different inclusion 
criteria may have contributed to a high KRAS mutation rate in the study. ${ }^{10}$

Our study primarily focused on extrovarian implants of serous borderline tumors/atypical proliferative serous tumors, therefore, majority of patients in our study had high stage disease. Among the 42 extraovarian tumor implants, 14 implants were associated with stage IIIC tumor (Table 3). 9 of 14 patients (64\%) with KRAS mutation detectable in their tumor implants had stage IIIC disease, while only 3 patients with stage IIA and IIB had KRAS mutation. Independent of the histological subtypes of implants, KRAS mutation is a significant prognostic indicator for tumor recurrence as the patients carrying KRAS mutation had a significantly higher recurrence rate than those without the mutation (71 vs $21 \%, P=0.0021$, log-rank test). A worse diseasespecific survival was also observed for patients with KRAS mutation positive implants compared with those without the mutation $(P=0.0104$, log-rank test) regardless of the histological subtype of implants. To our knowledge, this is the first report that KRAS mutation is found statistically associated with worse disease-free and disease-specific survival for patients of serous borderline tumors/atypical proliferative serous tumors with extraovarian tumor implants. Our data strongly indicate that KRAS mutation analysis may be a clinically useful marker for prediction of recurrence and survival of patients with extraovarian tumor implants of serous borderline tumor.

In several previous studies, GTT (G12V), GAT (G12D), and GCT (G12A) were found as common KRAS mutation variants in serous borderline tumors/atypical proliferative serous tumors and low-grade serous carcinoma. ${ }^{9,14}$ One recent study indicates that patients carrying KRAS G12V mutation (GTT mutation) have a significantly shorter overall survival than those with KRAS G12D mutation in recurrent low-grade serous carcinomas. ${ }^{9}$ KRAS G12V mutation has also been associated with unfavorable survival in colorectal and lung cancers. ${ }^{19,20}$ Consistently, the two patients with G12V mutation in the current study died of the disease. However, G12S (AGT mutation) instead of G12D was the most frequent mutation in extraovarian tumor implants in our study. Three of five patients carrying G12S also died of the disease, suggesting unfavorable impact of G12S mutation on patient survival. Biologically, an amino acid change from the wild-type glycine to serine residue-as a result of G12S mutation-is potentially relevant to the tumor biology as serine is one of the major biological phosphorylation sites in oncogenes, such as KRAS. Further investigations into larger cohorts of patients are needed to ascertain the biological implication of various KRAS mutations.

Previous data have suggested that $B R A F$ mutation is primarily found in serous borderline tumors/ atypical proliferative serous tumors without extraovarian involvement and in non-invasive implants.
$B R A F$ mutation is uncommon in invasive implants and low-grade serous carcinoma. Consistently, in the current study, BRAF V600E mutation was seen only in 1 of 22 non-invasive implants $(5 \%)$ and none of the 20 invasive implants harbored BRAF V600E mutation (0\%). Previous studies showed 23 to $48 \%$ of serous borderline tumors/atypical proliferative serous tumors carrying the $B R A F$ mutation while the rate dropped to 0 to $33 \%$ in low-grade serous carcinomas. ${ }^{4}$ Regarding BRAF mutation status in implants, one study found that 14 of $63(22 \%)$ noninvasive implants and none of 7 invasive implants had BRAF mutation by either PCR-Sanger sequencing or immunohistochemistry analysis. ${ }^{10}$ Similarly, none of 23 recurrent low-grade serous carcinoma had $B R A F$ mutation but 5 of 13 noncurrent cases had the mutation in another study. ${ }^{9}$

In conclusion, patients with invasive implants of serous borderline tumors/atypical proliferative serous tumors more frequently present at a higher disease stage, and incur a higher recurrence rate and a worse overall survival compared with those with non-invasive implants. KRAS mutation, but not $B R A F$ V600E mutation, is significantly associated with the presence of invasive implants of serous borderline tumors/atypical proliferative serous tumors. Regardless of the histological subtypes of the implants, KRAS mutation is a significant prognostic indicator for high risk of tumor recurrence and worse disease-specific survival. Future studies of large patient cohorts are important to establish the clinical validity of KRAS mutation testing for diagnostic and prognostic applications in patients with ovarian serous borderline tumor/atypical proliferative serous tumors.

\section{Disclosure/conflict of interest}

The authors declare no conflict of interest.

\section{References}

1 Krzystyniak J, Ceppi L, Dizon DS, et al. Epithelial ovarian cancer: the molecular genetics of epithelial ovarian cancer. Ann Oncol 2016;27:i4-i10.

2 McKenney JK, Gilks CB, Kalloger S, et al. Classification of extraovarian implants in patients with ovarian serous borderline tumors (tumors of low malignant potential) based on clinical outcome. Am J Surg Pathol 2016;40:1155-1164.

3 Seidman JD, Bell DA, Crum CP, et al. Tumours of the ovary: epithelial tumours-serous tumors. In:Kurman RJ, Carcangiu ML, Herrington CS, et al. eds. WHO Classification of Tumours of Female Reproductive Organs. 4th ed. International Agency for Research on Cancer (IARC) Press: Lyon, France, 2014;17-24.

4 Malpica A, Wong KK. The molecular pathology of ovarian serous borderline tumors. Ann Oncol 2016;27: i16-i19.

5 Hannibal CG, Vang R, Junge J, et al. A nationwide study of serous 'borderline' ovarian tumors in Denmark 
1978-2002: centralized pathology review and overall survival compared with the general population. Gynecol Oncol 2014;134:267-273.

6 Ahn G, Folkins AK, McKenney JK, et al. Low-grade serous carcinoma of the ovary: clinicopathologic analysis of 52 invasive cases and identification of a possible noninvasive intermediate lesion. Am J Surg Pathol 2016;40:1165-1176.

7 Jones S, Wang TL, Kurman RJ, et al. Low-grade serous carcinomas of the ovary contain very few point mutations. J Pathol 2012;226:413-420.

8 Singer G, Oldt R 3rd, Cohen Y, et al. Mutations in BRAF and KRAS characterize the development of low-grade ovarian serous carcinoma. J Natl Cancer Inst 2003;95: 484-486.

9 Tsang YT, Deavers MT, Sun CC, et al. KRAS (but not BRAF) mutations in ovarian serous borderline tumour are associated with recurrent low-grade serous carcinoma. J Pathol 2013;231:449-456.

10 Ardighieri L, Zeppernick F, Hannibal CG, et al. Mutational analysis of BRAF and KRAS in ovarian serous borderline (atypical proliferative) tumours and associated peritoneal implants. J Pathol 2014;232:16-22.

11 Bell KA, Smith Sehdev AE, Kurman RJ. Refined diagnostic criteria for implants associated with ovarian atypical proliferative serous tumors (borderline) and micropapillary serous carcinomas. Am J Surg Pathol 2001;25:419-432.

12 Zhong H, Liu Y, Talmor M, et al. Deparaffinization and lysis by hydrothermal pressure (pressure cooking) coupled with chaotropic salt column purification: a rapid and efficient method of DNA extraction from formalin-fixed paraffin-embedded tissue. Diagn Mol Pathol 2013;22:52-58.

13 Dillon DA, Johnson CC, Topazian MD, et al. The utility of Ki-ras mutation analysis in the cytologic diagnosis of pancreatobiliary neoplasma. Cancer J 2000;6:294-301.

14 Perincheri S, Hui P. KRAS mutation testing in clinical practice. Exp Rev Mol Diagn 2015;15:375-384.

15 Bell DA, Weinstock MA, Scully RE. Peritoneal implants of ovarian serous borderline tumors. Histologic features and prognosis. Cancer 1988;62:2212-2222.

16 McCaughey WT, Kirk ME, Lester W, et al. Peritoneal epithelial lesions associated with proliferative serous tumours of ovary. Histopathology 1984;8: 195-208.

17 Ho CL, Kurman RJ, Dehari R, et al. Mutations of BRAF and KRAS precede the development of ovarian serous borderline tumors. Cancer Res 2004;64:6915-6918.

18 Vang R, Hannibal CG, Junge J, et al. Long-term behavior of serous borderline tumors subdivided into atypical proliferative tumors and noninvasive low-grade carcinomas: a population-based clinicopathologic study of 942 cases. Am J Surg Pathol 2017;41:725-737.

19 Andreyev HJ, Norman AR, Cunningham D, et al. Kirsten ras mutations in patients with colorectal cancer: the 'RASCAL II' study. Br J Cancer 2001;85: 692-696.

20 Keohavong P, DeMichele MA, Melacrinos AC, et al. Detection of K-ras mutations in lung carcinomas: relationship to prognosis. Clin Cancer Res 1996;2: 411-418. 